\title{
Hypothalamic Overexpression of Neurosecretory Protein GL Leads to Obesity in Mice
}

Yuki Narimatsu ${ }^{\mathrm{a}}$, Keisuke Fukumura ${ }^{\mathrm{a}}$, Kenshiro Shikano, ${ }^{\mathrm{a}, \mathrm{b}}$, Eiko Iwakoshi-Ukena ${ }^{\mathrm{a}}$, Megumi

Furumitsu $^{\mathrm{a}}$, George E. Bentley ${ }^{\mathrm{c}}$, Lance J. Kriegsfeld ${ }^{\mathrm{d}}$ and Kazuyoshi Ukena ${ }^{\mathrm{a}^{*}}$

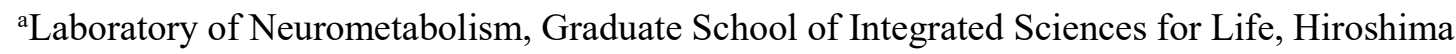
University, Higashi-Hiroshima, Hiroshima 739-8521, Japan

${ }^{b}$ Department of Neurophysiology, Faculty of Medicine, Oita University, Yufu, Oita 879-5593, Japan

${ }^{c}$ Department of Integrative Biology and the Helen Wills Neuroscience Institute, University of California, Berkeley, CA 94720-3140, USA

${ }^{\mathrm{d} D e p a r t m e n t}$ of Psychology, Integrative Biology, and the Helen Wills Neuroscience Institute, University of California, Berkeley, CA 94720-3140, USA

${ }^{*}$ Corresponding Author:

Kazuyoshi Ukena

Laboratory of Neurometabolism, Graduate School of Integrated Sciences for Life

Hiroshima University

Higashi-Hiroshima, Hiroshima 739-8521, Japan

E-mail: ukena@hiroshima-u.ac.jp

Short title: A novel mouse model of neurosecretory protein GL- induced obesity Keywords: Neurosecretory protein GL; Hypothalamus; Obesity; Animal model 


\begin{abstract}
Introduction: The mechanisms underlying obesity are not fully understood, necessitating the creation of novel animal models for investigation of metabolic disorders from the cellularmolecular to behavioral levels of analysis. We have previously found that neurosecretory protein GL (NPGL), a newly-identified hypothalamic neuropeptide, is involved in feeding behavior and fat accumulation in rats. Given the broad availability of genetic tools in mice, the present investigation sought to establish a mouse model of NPGL-induced obesity.

Methods: We overexpressed the NPGL-precursor gene ( $\mathrm{Npgl}$ ) in the hypothalamus using adeno-associated virus in C57BL/6J mice fed normal chow (NC) or a high-calorie diet (HCD). After 9 weeks of $\mathrm{Npgl}$ overexpression, we measured adipose tissues, muscle, and several organ masses in addition to food intake and body mass. To assess the effects of $\mathrm{Npgl}$ overexpression on peripheral tissues, we analyzed mRNA expression of lipid metabolism-related genes by quantitative RT-PCR.
\end{abstract}

Results: $\mathrm{Npgl}$ overexpression increased food intake, body mass, adipose tissues and liver masses, food efficiency, and circulating insulin levels under both $\mathrm{NC}$ and $\mathrm{HCD}$, resulting in obesity observable within 8 weeks. Furthermore, we observed fat accumulation in adipose tissues and liver. Additionally, mRNA expression of lipid metabolism-related factors was increased in white adipose tissue and the liver after $\mathrm{Npgl}$ overexpression.

Conclusion: Taken together, the present study suggests that NPGL is an endogenous obesogenic factor that acts within a short period of time in mice. As a result, this animal model can be widely applied to study the etiology of obesity from genes to behavior. 


\section{Introduction}

The number of obese patients worldwide has recently reached 700 million [1]. Obesity is associated with many serious diseases, such as type 2 diabetes mellitus, hypertension, and hyperlipidemia [2-5]. The imbalance between energy intake and energy expenditure causes fat accumulation and eventually obesity [6]. Therefore, further research on energy homeostasis is required to prevent and treat metabolic disease. Energy homeostasis is regulated by factors produced by the brain and peripheral tissues. The neuropeptides $\alpha$-melanocyte-stimulating hormone $(\alpha-\mathrm{MSH})$ - derived from proopiomelanocortin (POMC) - and neuropeptide Y (NPY) are known to be anorexigenic and orexigenic respectively. Both are produced in the hypothalamic arcuate nucleus (Arc) [7]. Agouti-related peptide (AgRP) is also produced by NPY-expressing neurons and inhibits the effect of $\alpha$-MSH by antagonistic binding to melanocortin receptor type 4 (MC4R) [8]. Ghrelin and leptin are other well-known energyrelated metabolic factors secreted by peripheral tissues. Ghrelin is an orexigenic factor secreted by the stomach, and it promotes feeding behavior by activating NPY/AgRP neurons [9]. Leptin is an anorexigenic hormone released from white adipose tissue (WAT) [10, 11]. Moreover, insulin accelerates fat accumulation by inhibiting lipolysis [12]. It is clear that many factors are involved in the regulation of energy intake, but this complex system is still not fully understood. Thus, the identification and functional analysis of novel factors is required for understanding whole body energy homeostasis.

We recently found a novel gene related to energy homeostasis in chickens, rats, mice, and humans [13-15]. The small secretory protein produced by the novel gene was named neurosecretory protein GL (NPGL) because the C-terminus amino acid sequence is Gly-Leu$\mathrm{NH}_{2}$ [13]. Analysis of the genome database revealed that NPGL is evolutionarily conserved throughout vertebrates [13]. To date, we have mainly investigated the functions and features of NPGL in rats and mice. Using morphological analysis, we found that NPGL is produced in the lateroposterior region of the Arc in mice, and NPGL-containing fibers project to several regions within the hypothalamus [15].

We recently showed that NPGL regulates feeding and fat accumulation in rodents. In rats, a 13-day chronic intracerebroventricular (i.c.v.) infusion of NPGL increased the WAT mass 
without changing body mass and modestly stimulated food intake under a high-calorie diet (HCD) [14]. Analysis of adeno-associated virus (AAV)-driven Npgl overexpression in rats for 6 weeks had more marked effects on feeding behavior and lipogenesis than the 13-day chronic infusion [14]. Similarly, 13-day chronic i.c.v. infusion of NPGL stimulated feeding behavior and mass gain due to fat accumulation in mice fed a HCD [16]. However, the induction of abnormally high body mass was not a characteristic result of NPGL treatment in these studies, leaving the possibility that NPGL induces obesity as an open question. In general, creation of diet-induced obesity (DIO) in rodents is costly and time-consuming, typically requiring 16-20 weeks [17-19]. Therefore, the discovery of a more efficient way to generate an animal model of obesity would be useful for studies aiming to elucidate the mechanism of energy metabolism regulation.

The aim of the present study was to determine the impact of NPGL on obesity in a widelyused animal model with broad foundational data and molecular-genetic tools available. We found that overexpression of the NPGL-precursor gene ( $\mathrm{Npgl}$ ) in the mouse hypothalamus elicited obesity within 8 weeks under a HCD. The present findings report the effects of $\mathrm{Npgl}$ overexpression on food intake, body mass, body composition, blood parameters, and the expression of lipid metabolism-related factors in mice fed normal chow (NC) or a HCD during the development of obesity.

\section{Materials and Methods}

\section{Animals}

Male C57BL/6J mice (7 weeks old) were purchased from Nihon SLC (Hamamatsu, Japan) and housed under standard conditions $\left(25 \pm 1{ }^{\circ} \mathrm{C}\right.$ under a 12-h light/12-h dark cycle) with ad libitum access to water and NC (CE-2; CLEA Japan, Tokyo, Japan) or a HCD (32\% of calories from fat $/ 20 \%$ of calories from sucrose, D14050401; Research Diets, New Brunswick, NJ). Animal surgery was performed under isoflurane anesthesia. 
We followed a previously reported method to generate the overexpression AAV [14]. The full-length open reading frame of mouse $\mathrm{Npgl}$ was amplified from cDNA of the mediobasal hypothalamus and inserted into the pAAV-IRES-GFP expression vector (Cell Biolabs, San Diego, CA). The primers for mouse NPGL were 5'-

CGATCGATACCATGGCTGATCCTGGGC-3' for the sense primer and 5'CGGAATTCTTATTTTCTCTTTACTTCCAGC-3' for the antisense primer.

AAV-based vectors AAV-DJ/8-NPGL-IRES-GFP for NPGL (AAV-NPGL) and AAV-DJ/8IRES-GFP for control (AAV-CTL) as shown in supplementary Fig. S1a were produced in 293AAV cells (Cat\# AAV-100; Cell Biolabs) using the AAV-DJ/8 Helper Free Packaging System containing pAAV-DJ/8 and pHelper plasmids (Cell Biolabs). The triple plasmids (AAV-DJ/8-NPGL-IRES-GFP or AAV-DJ/8-IRES-GFP, pAAV-DJ/8, and pHelper) were mixed with the polyethylenimine MAX transfection reagent (PEI-MAX; Polysciences, Warrington, PA). The mixture was diluted with Opti-MEM I medium (Life Technologies, Carlsbad, CA) and added to 293AAV cells in 150-mm cell culture dishes. Transfected cells were cultured in DMEM containing 10\% fetal bovine serum.

For the purification of AAV-based vectors, 3 days after transfection, the cells and supernatants were harvested and purified using chloroform and were condensed using Amicon Ultra-4 Centrifugal Filter Devices (100K MWCO; Merck Millipore, Billerica, MA).

For AAV titration, $1 \mu \mathrm{L}$ of AAV solution was treated with RQ1 DNase (Promega, Madison, WI) according to the manufacturer's instructions. Virus titers were determined by quantitative PCR with EGFP primer pairs. The primers for EGFP were 5'ACCACTACCTGAGCACCCAGTC-3' for the sense primer and 5'GTCCATGCCGAGAGTGATCC-3' for the antisense primer. After titration, the AAV-based vectors were prepared at a concentration of $1 \times 10^{9}$ particles $/ \mu \mathrm{L}$ and stored at $-80^{\circ} \mathrm{C}$ until use.

\section{Npgl Overexpression}

Mice were divided into two groups according to each diet (NC or HCD). For Npgl overexpression, mice were bilaterally injected with $0.5 \mu \mathrm{L} /$ site $\left(5.0 \times 10^{8}\right.$ particles $/$ site $)$ of AAV-based vectors (AAV-NPGL or AAV-CTL) using a Neuros Syringe (7001 KH; Hamilton, 
Reno, NV) into the mediobasal hypothalamic region with the coordinates $2.2 \mathrm{~mm}$ caudal to the bregma, $0.25 \mathrm{~mm}$ lateral to the midline, and $5.8 \mathrm{~mm}$ ventral to the skull surface.

$\mathrm{Npgl}$ overexpression was maintained for 9 weeks in mice fed NC or a HCD. $N p g l$ overexpression was confirmed by quantitative RT-PCR at the endpoint. Food intake and body mass were measured weekly. Food efficiency (g/kcal) was calculated as body mass gain (g)/ cumulative food intake (kcal) [20]. Body composition and serum parameters were measured at the end of $\mathrm{Npgl}$ overexpression.

\section{Quantitative RT-PCR}

The inguinal WAT (iWAT) and liver were dissected from mice and snap frozen in liquid nitrogen for RNA processing. Total RNA was extracted using QIAzol lysis reagent for the iWAT (QIAGEN, Venlo, Netherlands) or TRIzol reagent for the liver (Life Technologies) in accordance with the manufacturer's instructions. First-strand cDNA was synthesized from total RNA using a ReverTra Ace kit (TOYOBO, Osaka, Japan).

The abbreviations for genes and sequences of primers used in this study are listed in Table 1 and Table 2, respectively. PCR amplifications were conducted with THUNDERBIRD SYBR qPCR Mix (TOYOBO) using the following conditions: $95^{\circ} \mathrm{C}$ for $20 \mathrm{~s}$, followed by 40 cycles each consisting of $95^{\circ} \mathrm{C}$ for $3 \mathrm{~s}$, and $60^{\circ} \mathrm{C}$ for $30 \mathrm{~s}$. The PCR products in each cycle were monitored using a Bio-Rad CFX Connect (Bio-Rad Laboratories, Hercules, CA). Relative quantification of each gene was determined by the $2^{-\Delta \Delta \mathrm{Ct}}$ method using ribosomal protein S18 (Rps18) for the iWAT, or $\beta$-actin (Actb) for the liver as an internal control [21].

\section{Fatty Acid Analysis}

For analysis of endogenous stearoyl-CoA desaturase 1 (SCD1) activity in the iWAT, lipids were extracted according to a previously described method [22]. The iWAT (50 mg) was extracted with $500 \mu \mathrm{L}$ of chloroform:methanol (2:1) using a bead crusher ( $\mu \mathrm{T}-12$; TAITEC, Saitama, Japan), and $125 \mu \mathrm{L}$ of distilled water was added and mixed by inversion. After incubation for $30 \mathrm{~min}$, the sample was centrifuged at $3000 \times \mathrm{g}$, and the lower organic phase was collected and evaporated. Extracted fatty acids were methylated using a Fatty Acid Methylation 
Kit (Nacalai Tesque, Kyoto, Japan) and purified using a Fatty Acid Methyl Ester Purification Kit (Nacalai Tesque). The eluted solution was evaporated to dryness and kept at $-20^{\circ} \mathrm{C}$. The residues were solubilized in hexane, and fatty acids were identified by gas chromatographymass spectroscopy (GC-MS) (JMS-T100 GCV; JEOL, Tokyo, Japan). SCD1 activity was estimated as the oleate-to-stearate ratio (18:1/18:0) and palmitoleate-to-palmitate ratio (16:1/16:0). The 16:1/16:0 ratio seems to be a better indicator of endogenous SCD1 activity than the 18:1/18:0 ratio [23]. The de novo lipogenesis index was calculated from the palmiticto-linoleic acid ratio $(16: 0 / 18: 2 n-6)[24,25]$.

\section{Immunohistochemistry}

The mediobasal hypothalamus was injected with AAV-CTL or AAV-NPGL, as described above. Four weeks later, the brain tissues were sectioned into $20-\mu \mathrm{m}$-thick slices with a cryostat at $-20^{\circ} \mathrm{C}$ following cryoprotection and freezing. The sections were incubated in blocking buffer ( $1 \%$ bovine serum albumin, $1 \%$ normal donkey serum, and $0.3 \%$ Triton X-100 in $10 \mathrm{mM}$ phosphate-buffered saline) for $1 \mathrm{~h}$ at room temperature before incubation with a rabbit antibody against NPGL (1:100 dilution in blocking buffer), overnight at $4^{\circ} \mathrm{C}$. Cy3-conjugated donkey anti-rabbit IgG (1:500 dilution, 711-165-152; Jackson ImmunoResearch Laboratories, West Grove, PA) was used as the secondary antibody. Immunoreactive labeling was observed using a microscope (Eclipse E600; Nikon, Tokyo, Japan).

\section{Hematoxylin and Eosin Staining}

The iWAT was soaked in 4\% paraformaldehyde at the endpoint of $\mathrm{Npgl}$ overexpression, embedded in paraffin, and sectioned to a thickness of $8 \mu \mathrm{m}$ with a microtome. The sections were then air-dried and deparaffinized in a graded alcohol series. The nucleus and cytoplasm were stained with hematoxylin and eosin ( 5 min for each stain), and the sections were washed in tap water. After dehydration in a graded alcohol series and clearing with xylene, the sections were mounted on slides and examined under a microscope.

\section{Oil Red O Staining}


To detect fat accumulation in the liver, the liver was fixed in 4\% paraformaldehyde and sliced into $10-\mu \mathrm{m}$-thick sections. Sections were air-dried, rinsed with $60 \%$ isopropanol, stained with Oil Red O solution for $15 \mathrm{~min}$ at $37^{\circ} \mathrm{C}$, and rinsed with $60 \%$ isopropanol. Nuclei were counterstained with hematoxylin for $5 \mathrm{~min}$, and sections were then washed in tap water.

Coverslips were applied using an aqueous mounting medium, and microscopic examination was performed using a microscope.

\section{Serum Biochemical Analysis}

Serum levels of glucose, lipids, and insulin were measured using appropriate equipment, reagents, and kits. The GLUCOCARD G+ meter was used to measure glucose content (Arkray, Kyoto, Japan). The NEFA C-Test Wako (Wako Pure Chemical Industries, Osaka, Japan) was used to measure free fatty acid levels. Triglyceride E-Test Wako (Wako Pure Chemical Industries) was used to measure triglyceride levels and the Cholesterol E-Test Wako (Wako Pure Chemical Industries) was used to assess cholesterol content. The Rebis Insulin-mouse T ELISA kit (Shibayagi, Gunma, Japan) was used to measure insulin levels.

\section{Statistical Analysis}

Group differences between AAV-NPGL and AAV-CTL injected animals were assessed using Student's $t$-test or Welch's $t$-test. $P$ values of $<0.05$ were considered significant.

\section{Results}

Effects of NPGL-precursor Gene Overexpression on Food Intake, Body Mass, and Food

\section{Efficiency}

AAV-induced overexpression in the mediobasal hypothalamus was confirmed by immunohistochemistry (Supplementary Fig. S1b). Chronic (9 week) $\mathrm{Npgl}$ overexpression in the hypothalamus significantly increased cumulative food intake and body mass in mice fed NC and a HCD relative to controls (Fig. 1a-d). 
To determine whether the increase in body mass was caused by food intake, we next calculated food efficiency, which is a measure of how much mass is gained per unit of food intake [20]. Food efficiency was markedly increased by $\mathrm{Npgl}$ overexpression in mice fed NC and those fed a HCD (Fig. 1e). Npgl-overexpressing mice fed a HCD were visibly obese, as compared to the AAV-based control vector-injected mice (Fig. 1f).

\section{Effects of NPGL-precursor Gene Overexpression on Body Composition and Serum Parameters}

To address the cause of $\mathrm{Npgl}$ overexpression-induced body mass increase, we measured the masses of adipose tissues, muscle, and several organs (Figs. 2 and 3). In mice fed NC, the masses of WAT and interscapular brown adipose tissue (BAT) were significantly higher in Npgl-overexpressing mice relative to control mice (Fig. 2a, d). Under a HCD, WAT mass and adipocyte size were considerably increased in Npgl-overexpressing mice (Fig. 2a-c), whereas there was no difference in the BAT mass in Npgl-overexpressing mice (Fig. 2d).

While the mass of WAT increased markedly, the mass of the gastrocnemius muscle was not different in $\mathrm{Npgl}$-overexpressing mice in either diet condition (Fig. 3a). With regard to peripheral organs, the mass of the liver was increased relative to controls by $\mathrm{Npgl}$ overexpression under both feeding conditions (Fig. 3b). In addition, $\mathrm{Npgl}$ overexpression induced a pronounced whitening of the liver in mice fed a HCD (Fig. 3c). To explore the underlying cause for the whitening of the liver, we performed a histological examination of lipid deposition in the liver. Oil Red O staining revealed that lipid droplets were increased in the liver in mice with $\mathrm{Npgl}$ overexpression (Fig. 3c). The masses of the testis and heart were not changed

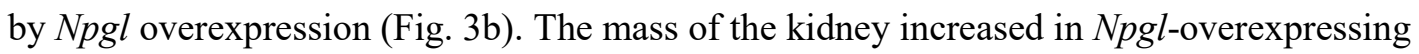
mice fed NC, whereas there was no effect when these mice were fed a HCD (Fig. 3b).

Fat accumulation can cause hyperglycemia and hyperlipidemia [5]. To reveal the effects of $\mathrm{Npgl}$ overexpression on blood parameters, we measured serum levels of glucose, insulin, and lipids. $N p g l$ overexpression increased serum levels of insulin and cholesterol under both feeding conditions, whereas serum levels of glucose and free fatty acids were unaffected (Fig. 4a, b, d, e). Moreover, the serum triglyceride level was increased by $\mathrm{Npgl}$ overexpression in mice fed NC (Fig. 4c). 


\section{Effects of NPGL-precursor Gene Overexpression on mRNA Expression and Endogenous}

\section{Activity of Lipid Metabolism-related Genes}

We detected fat accumulation in the WAT and liver as a result of $\mathrm{Npgl}$ overexpression, so we analyzed the mRNA expression of lipid metabolism-related genes by quantitative RT-PCR in the iWAT and the livers of mice fed NC or a HCD. The following genes were analyzed: acetyl-CoA carboxylase (Acc), fatty acid synthase ( $F a s), S c d 1$, and glycerol-3-phosphate acyltransferase 1 (Gpat1) as genes encoding lipogenic enzymes; carbohydrate-responsive element-binding protein $\alpha($ Chrebp $\alpha)$ as a lipogenic transcription factor; carnitine palmitoyltransferase 1a (Cptla), adipose triglyceride lipase (Atgl), hormone-sensitive lipase $(H s l)$, and fibroblast growth factor $21(F g f 21)$ as genes encoding lipolytic enzymes; glyceraldehyde-3-phosphate dehydrogenase (Gapdh) as a carbohydrate metabolism enzyme gene; solute carrier family 2 member 4 and 2 (Slc2a4, Slc2a2) as glucose transporters; cluster of differentiation $36(\mathrm{Cd} 36)$ as a fatty acid transporter; peroxisome proliferator-activated receptor $\alpha($ Ppar $\alpha)$ and $\gamma($ Ppar $\gamma)$ as genes encoding lipid-activated transcription factors; peroxisome proliferator-activated receptor $\gamma$ coactivator $1 \alpha(P g c l \alpha)$ as a thermogenic regulator gene; tumor necrosis factor $\alpha(\operatorname{Tnf} \alpha)$ as an inflammatory cytokine gene; and adiponectin (Adipoq) as an antiinflammatory agent-encoding gene. Under NC condition, mRNA expression levels of Gpat1, Chrebpa, Cpt1a, Atgl, Hsl, Slc2a4, Cd36, Ppary, and Adipoq were increased by Npgl overexpression in the iWAT (Fig. 5a). In contrast, the mRNA expression level of Tnf $\alpha$ was decreased (Fig. 5a). Under a HCD with $\mathrm{Npgl}$ overexpression, mRNA expression levels of Chrebp $\alpha$ were upregulated and those of $\operatorname{Tnf} \alpha$ were downregulated relative to controls, similar to the observations in mice fed $\mathrm{NC}$ with $\mathrm{Npgl}$ overexpression (Fig. 5a).

In the liver, mRNA expression levels of Gapdh and Cd36 were upregulated in Npgloverexpressing mice fed NC. Under a HCD, mRNA expression levels of Gpat1, Fgf21, Gapdh, and $C d 36$ were increased by $N p g l$ overexpression. In contrast, mRNA expression levels of Chrebpa, Cpt1a, and Ppara were decreased (Fig. 5b).

To confirm the activity of lipogenic factors in the iWAT, we measured the fatty acid ratio using GC-MS. The fatty acid ratios of palmitoleate-to-palmitate (16:1/16:0) and of oleate-to- 
stearate (18:1/18:0) indicate increased enzymatic activity of SCD1 [23]. The ratio 16:0/18:2n-6 is an index of de novo lipogenesis [24,25]. The fatty acid ratio of 16:1/16:0 was significantly increased in the iWAT of $N p g l$-overexpressing mice fed NC, although the 18:1/18:0 and 16:0/18:2n-6 ratios remained unchanged (Fig. 6a-c). This result indicates activation of SCD1 by Npgl overexpression in the iWAT under NC condition. Under the HCD condition, these ratios did not change in the iWAT upon Npgl overexpression (Fig. 6a-c).

\section{Discussion}

We have demonstrated that NPGL is involved in energy homeostasis in vertebrates, including birds and rodents [13-16, 26, 27]. In particular, we recently showed that $\mathrm{Npgl}$ overexpression modestly stimulated feeding behavior and fat accumulation in rats [14]. However, whether NPGL causes obesity across species has not previously been clearly elucidated. In the present study, we found that $\mathrm{Npgl}$ overexpression in mice induced a remarkable increase in food intake, body mass, and fat mass under both $\mathrm{NC}$ and $\mathrm{HCD}$ conditions. Therefore, these data indicate that NPGL stimulates fat accumulation in WAT very rapidly, resulting in obesity within 8 weeks in mice.

This difference in the effects of $\mathrm{Npgl}$ overexpression on body mass change between mice and rats might be a result of species differences in metabolism. Wistar rats were used in the previous study [14], while C57BL/6J mice, which are susceptible to obesity [28], were used in this study. Another possibility may be the difference in the amount of food intake between rats and mice. Although cumulative food intake was slightly increased at the end point in rats fed $\mathrm{NC}$ [14], feeding was markedly increased in mice from the early during the treatment period and for the remainder of this experiment in mice (Fig. 1a, b). Using morphological analysis, we previously found that NPGL-containing fibers innervate neurons expressing POMC, a precursor of potent anorexigenic $\alpha-\mathrm{MSH}$, in the mouse hypothalamus [15]. However, this finding was not observed in rats (unpublished observation). Thus, the orexigenic activity of NPGL due to suppression of the activity of POMC neurons in mice is likely higher than that in rats. Future 
electrophysiological studies are needed to elucidate the efficacy of NPGL in terms of negative modulation of POMC neurons in mice.

The present study revealed that food efficiency was increased by $\mathrm{Npgl}$ overexpression (Fig. 1e). This result suggests that NPGL-induced fat accumulation is caused not only by energy intake, but also by other factors. Our previous study showed that chronic i.c.v. infusion of NPGL for 13 days reduced energy expenditure and locomotor activity in mice [16]. On the other hand, many studies have demonstrated that obesity resulting from fat accumulation is closely related to inflammation in the WAT $[17,29,30]$. Inflammation in the WAT is mainly regulated by TNF $\alpha$, an inflammatory cytokine and adiponectin, an anti-inflammatory cytokine [29]. TNF $\alpha$ directly induces lipolysis in adipocytes [31]. Moreover, adiponectin improves insulin sensitivity in the WAT [32]. The present study demonstrated that the mRNA expression of $\operatorname{Tnf} \alpha$ was decreased, while that of Adipoq was increased in iWAT following Npgl overexpression (Fig. 5a). Taken together, these results suggest that NPGL suppresses inflammation in adipose tissues, resulting in improved insulin sensitivity and fat accumulation. Further studies are required to clarify the involvement of NPGL in the inflammatory response in the adipose tissue and insulin sensitivity.

Quantitative RT-PCR showed that $\mathrm{Npgl}$ overexpression increased mRNA expression of factors involved in lipid metabolism in iWAT, especially under the NC condition (Fig. 5a). This result is not surprising as previous findings establish that lipid metabolism is responsive to nutritional conditions [33]. Docosahexaenoic acid, one of the polyunsaturated fatty acids,

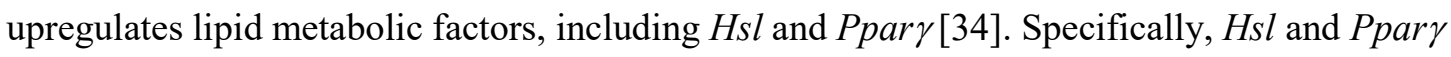
mRNA expression were upregulated by $\mathrm{Npgl}$ overexpression in the iWAT of mice fed NC, but were unchanged under the HCD condition (Fig. 5a). Therefore, our study suggests that intake of HCD may mitigate the effects of NPGL on lipid metabolism. In addition, $N p g l$ overexpression upregulated mRNA expression for factors involved in both lipogenesis and lipolysis; also activating SCD1 (Fig. 5a, 6). Lipolysis is regulated by several factors, including ATGL and HSL [35]. In particular, HSL is thought to be the rate-limiting enzyme of lipolysis, and the enzyme is activated by phosphorylation $[36,37]$. In DIO mice, HSL protein levels were highly similar to those in control lean mice, although the level of HSL phosphorylation was 
significantly decreased [38]. Furthermore, insulin stimulates the degradation of cAMP through activation of phosphodiesterase-3B (PDE-3B), suppressing HSL activation [37]. Indeed, we observed an increase in serum insulin levels as a result of $\mathrm{Npgl}$ overexpression in mice (Fig. 4b). Therefore, it is possible that HSL might not be activated by phosphorylation despite the abundance of $H s l$ mRNA. Conversely, the upregulation of mRNA expression related to lipolysis might be a result of feedback control due to fat accumulation. Further studies are needed to elucidate the effects of NPGL on lipolysis and lipogenesis to reveal the mechanism of fat accumulation in these mice.

$\mathrm{Npgl}$ overexpression increased liver mass in mice under NC and HCD conditions (Fig. 3b). A previous study reported that elevated insulin stimulates fat accumulation in the liver [39]. In agreement with this finding, we observed increases in serum insulin upon $\mathrm{Npgl}$ overexpression, as described above (Fig. 4b). Moreover, NPY, which is an orexigenic peptide like NPGL, regulates insulin secretion in rodents [40]. Therefore, the present study suggests that NPGL controls insulin secretion and triggers fat accumulation in the liver. In addition, a previous report revealed that fatty liver leads to hyperlipidemia, including hypercholesteremia and hypertriglyceridemia [41]. It is possible that the fatty liver in mice fed NC and HCD was induced by hyperinsulinemia, resulting in hyperlipidemia (Fig. 4b, c, e). Moreover, despite hyperinsulinemia and hyperlipidemia, blood glucose levels were not changed by $\mathrm{Npgl}$ overexpression (Fig. 4a, b, c, e). Therefore, NPGL may induce "metabolically healthy obesity", rather than obesity in the DIO model. This hypothesis is supported by the anti-inflammatory effects of $\mathrm{Npgl}$ overexpression in the WAT, as described above. Fat accumulation in the liver without excessive alcohol consumption is defined as nonalcoholic fatty liver disease (NAFLD) [42]. Most patients with NAFLD only exhibit simple steatosis [42]. Based on our data in mice, NPGL may also induce simple steatosis rather than nonalcoholic steatohepatitis (NASH). Further investigations are needed to clarify the effects of NPGL on insulin secretion, blood glucose homeostasis, and steatosis in the liver.

In conclusion, $\mathrm{Npgl}$ overexpression in mice enhances feeding behavior, fat accumulation, and secretion of insulin, rapidly resulting in obesity as compared to the DIO animal model that relies on a high-fat diet. Because Npgl overexpression maintains steady-state levels of blood 
glucose even in obesity, it may be a useful tool for creating a novel animal model of obesity compared to models induced by overeating, such as $o b / o b$ mice. Hence, progress in research on NPGL will contribute to a new approach in research on obesity.

\section{Acknowledgements}

We are grateful to Shiki Okamoto for discussion, and Takaya Saito and Atsuki Kadota for experimental support.

\section{Statement of Ethics}

All animal experiments were performed according to the Guide for the Care and Use of Laboratory Animals prepared by Hiroshima University (Higashi-Hiroshima, Japan), and these procedures were approved by the Institutional Animal Care and Use Committee of Hiroshima University (permit numbers: C11-2, C13-12, and C13-17).

\section{Conflict of Interest Statement}

The authors declare that no competing interests exist.

\section{Funding Sources}

This work was supported by JSPS KAKENHI Grants (JP15KK0259, JP18K19743, JP19H03258, and JP20K21760 to K.U., JP20K22741 to K.F., and JP19K06768 to E.I.-U.), the Mishima Kaiun Memorial Foundation (K.U. and E.I.-U.), the Urakami Foundation for Food and Food Culture Promotion (K.U. and E.I.-U.), the Takeda Science Foundation (K.U.), the Shiseido Female Researcher Science Grant (E.I.-U.), the Uehara Memorial Foundation (K.U.), and the ONO Medical Research Foundation (K.U.).

\section{Author Contributions}

Conceptualization, Y.N. and K.U.; methodology, Y.N., K.F., K.S., E.I.-U., and M.F.; investigation, Y.N., K.F., K.S., E.I.-U., M.F., G.E.B., L.J.K, and K.U.; writing—original draft preparation, Y.N., and K.F.; writing - review and editing, Y.N., K.F., G.E.B., L.J.K., and K.U.; 
bioRxiv preprint doi: https://doi.org/10.1101/2021.03.01.433475; this version posted March 2, 2021. The copyright holder for this preprint (which was not certified by peer review) is the author/funder. All rights reserved. No reuse allowed without permission.

visualization, Y.N.; project administration, K.U.; funding acquisition, K.F., E.I.-U., and K.U. All authors have read and agreed to the published version of the manuscript. 


\section{References}

1 Afshin A, Forouzanfar MH, Reitsma MB, Sur P, Estep K, Lee A, et al. Health effects of overweight and obesity in 195 countries over 25 years. N Engl J Med. 2017 Jul;377(1):13-27.

2 Jung U, Choi M-S. Obesity and its metabolic complications: The role of adipokines and the relationship between obesity, inflammation, insulin resistance, dyslipidemia and nonalcoholic fatty liver disease. Int J Mol Sci. 2014 Apr;15(4):6184-223.

3 Malone JI, Hansen BC. Does obesity cause type 2 diabetes mellitus (T2DM)? Or is it the opposite? Pediatr Diabetes. 2019 Feb;20(1):5-9.

4 Seravalle G, Grassi G. Obesity and hypertension. Pharmacol Res. 2017 Aug;122:1-7.

5 Sullivan PW, Ghushchyan VH, Ben-Joseph R. The impact of obesity on diabetes, hyperlipidemia and hypertension in the United States. Qual Life Res. 2008 Oct;17(8):1063-71.

6 Faria SL, Faria OP, Menezes CS, De Gouvêa HR, De Almeida Cardeal M. Metabolic profile of clinically severe obese patients. Obes Surg. 2012 Aug;22(8):1257-62.

7 Schwartz MW, Woods SC, Porte D, Seeley RJ, Baskin DG. Central nervous system control of food intake. Nature. 2000 Apr;404(6778):661-71.

8 Wilson BD, Bagnol D, Kaelin CB, Ollmann MM, Gantz I, Watson SJ, et al. Physiological and anatomical circuitry between agouti-related protein and leptin signaling. Endocrinology. 1999 May;140(5):2387-97.

9 Nakazato M, Murakami N, Date Y, Kojima M, Matsuo H, Kangawa K, et al. A role for ghrelin in the central regulation of feeding. Nature. 2001 Jan;409(6817):194-8.

10 Zhang Y, Proenca R, Maffei M, Barone M, Leopold L, Friedman JM. Positional cloning of the mouse obese gene and its human homologue. Nature. 1994 Dec;372(6505):42532.

11 Park HK, Ahima RS. Physiology of leptin: energy homeostasis, neuroendocrine function and metabolism. Metabolism. 2014 Jan;64(1):24-34. 
12 Koch L, Wunderlich FT, Seibler J, Könner AC, Hampel B, Irlenbusch S, et al. Central insulin action regulates peripheral glucose and fat metabolism in mice. J Clin Invest. 2008 Jun; 118(6):2132-47.

13 Ukena K, Iwakoshi-Ukena E, Taniuchi S, Bessho Y, Maejima S, Masuda K, et al. Identification of a cDNA encoding a novel small secretory protein, neurosecretory protein GL, in the chicken hypothalamic infundibulum. Biochem Biophys Res Commun. 2014 Mar;446(1):298-303.

14 Iwakoshi-Ukena E, Shikano K, Kondo K, Taniuchi S, Furumitsu M, Ochi Y, et al. Neurosecretory protein GL stimulates food intake, de novo lipogenesis, and onset of obesity. eLife. 2017 Aug;6:e28527. DOI: 10.7554/eLife.28527

15 Matsuura D, Shikano K, Saito T, Iwakoshi-Ukena E, Furumitsu M, Ochi Y, et al. Neurosecretory protein GL, a hypothalamic small secretory protein, participates in energy homeostasis in male mice. Endocrinology. 2017 May;158(5):1120-9.

16 Shikano K, Iwakoshi-Ukena E, Saito T, Narimatsu Y, Kadota A, Furumitsu M, et al. Neurosecretory protein GL induces fat accumulation in mice. J Endocrinol. 2020 Jan;244(1):1-12.

17 van der Heijden RA, Sheedfar F, Morrison MC, Hommelberg PPH, Kor D, Kloosterhuis NJ, et al. High-fat diet induced obesity primes inflammation in adipose tissue prior to liver in C57BL/6j mice. Aging (Albany NY). 2015 Apr;7(4):256-68.

18 Berger S, Pho H, Fleury-Curado T, Bevans-Fonti S, Younas H, Shin MK, et al. Intranasal leptin relieves sleep-disordered breathing in mice with diet-induced obesity. Am J Respir Crit Care Med. 2019 Mar;199(6):773-83.

19 Zheng J, Zhang J, Guo Y, Yang H, Lin A, Hu B, et al. Improvement on metabolic syndrome in high fat diet-induced obese mice through modulation of gut microbiota by sangguayin decoction. J Ethnopharmacol. 2020 Jan;246:112225.

20 Yang QQ, Suen PK, Zhang CQ, Mak WS, Gu MH, Liu QQ, et al. Improved growth performance, food efficiency, and lysine availability in growing rats fed with lysinebiofortified rice. Sci Rep. 2017 May;7(1):1389. 
21 Livak KJ, Schmittgen TD. Analysis of relative gene expression data using real-time quantitative PCR and the $2^{-\Delta \Delta C \mathrm{~T}}$ method. Methods. 2001 Dec;25(4):402-8.

22 Folch J, Lees M, Sloane Stanley G. A simple method for the isolation and purification of total lipides from animal tissues. 1957 May;226(1):497-509.

23 Sampath H, Ntambi \& J. Role of stearoyl-CoA desaturase in human metabolic disease. Future Lipidol. 2008;3(2):163-73.

24 Hudgins LC, Hellerstein M, Seidman C, Neese R, Diakun J, Hirsch J. Human fatty acid synthesis is stimulated by a eucaloric low fat, high carbohydrate diet. J Clin Invest. 1996 May;97(9):2081-91.

25 Rosqvist F, McNeil CA, Pramfalk C, Parry SA, Low WS, Cornfield T, et al. Fasting hepatic de novo lipogenesis is not reliably assessed using circulating fatty acid markers. Am J Clin Nutr. 2019 Feb;109(2):260-8.

26 Shikano K, Kato M, Iwakoshi-Ukena E, Furumitsu M, Matsuura D, Masuda K, et al. Effects of chronic intracerebroventricular infusion of neurosecretory protein GL on body mass and food and water intake in chicks. Gen Comp Endocrinol. 2018 Jan;256:37-42.

27 Shikano K, Iwakoshi-Ukena E, Kato M, Furumitsu M, Bentley GE, Kriegsfeld LJ, et al. Neurosecretory protein GL induces fat accumulation in chicks. Front Endocrinol (Lausanne). 2019 Jun;10:392.

28 Surwit RS, Kuhn CM, Cochrane C, McCubbin JA, Feinglos MN. Diet-induced type II diabetes in C57BL/6J mice. Diabetes. 1988 Sep;37(9):1163-7.

29 Stolarczyk E. Adipose tissue inflammation in obesity: a metabolic or immune response? Curr Opin Pharmacol. 2017 Dec;37:35-40.

30 Saltiel AR, Olefsky JM. Inflammatory mechanisms linking obesity and metabolic disease. J Clin Invest. 2017 Jan;127(1):1-4.

31 Suganami T, Nishida J, Ogawa Y. A paracrine loop between adipocytes and macrophages aggravates inflammatory changes: role of free fatty acids and tumor necrosis factor $\alpha$. Arterioscler Thromb Vasc Biol. 2005 Oct;25(10):2062-8. 
Zheng F, Zhang S, Lu W, Wu F, Yin X, Yu D, et al. Regulation of insulin resistance and adiponectin signaling in adipose tissue by liver $\mathrm{X}$ receptor activation highlights a crosstalk with PPAR $\gamma$. PLoS One. 2014 Jun;9(6):e101269.

33 Kersten S. Mechanisms of nutritional and hormonal regulation of lipogenesis. EMBO Rep. 2001 Apr;2(4):282-6.

34 Sun C, Wei ZW, Li Y. DHA regulates lipogenesis and lipolysis genes in mice adipose and liver. Mol Biol Rep. 2011 Feb;38(2):731-7.

35 Duncan RE, Ahmadian M, Jaworski K, Sarkadi-Nagy E, Sul HS. Regulation of lipolysis in adipocytes. Annu Rev Nutr. 2007 Feb;27:79-101.

36 Anthonsen MW, Rönnstrand L, Wernstedt C, Degerman E, Holm C. Identification of novel phosphorylation sites in hormone-sensitive lipase that are phosphorylated in response to isoproterenol and govern activation properties in vitro. J Biol Chem. 1998 Jan;273(1):215-21.

37 Frühbeck G, Méndez-Giménez L, Fernández-Formoso JA, Fernández S, Rodríguez A. Regulation of adipocyte lipolysis. Nutr Res Rev. 2014 Jun;27(1):63-93.

38 Kinney BP, Qiao L, LeVaugh JM, Shao J. B56 $\alpha$ /protein phosphatase 2A inhibits adipose lipolysis in high-fat diet-induced obese mice. Endocrinology. 2010 Aug;151(8):3624-32.

39 Horton JD, Goldstein JL, Brown MS. SREBPs: activators of the complete program of cholesterol and fatty acid synthesis in the liver. J Clin Invest. 2002 May;109(9):1125-31.

40 Moltz JH, McDonald JK. Neuropeptide Y: direct and indirect action on insulin secretion in the rat. Peptides. 1985 Nov-Dec;6(6):1155-9.

41 Grundy SM. Metabolic complications of obesity. Endocrine. 2000 Oct;13(2):155-65.

42 Buzzetti E, Pinzani M, Tsochatzis EA. The multiple-hit pathogenesis of non-alcoholic fatty liver disease (NAFLD). Metabolism. 2016 Aug;65(8):1038-48. 


\section{Figure legends}

\section{Fig. 1.}

Effects of $\mathrm{Npgl} \mathrm{overexpression} \mathrm{on} \mathrm{food} \mathrm{intake,} \mathrm{body} \mathrm{mass,} \mathrm{and} \mathrm{food} \mathrm{efficiency.} \mathrm{The} \mathrm{panels}$ show the data obtained by injection of the AAV-CTL or the AAV-NPGL in mice fed NC or a HCD for 9 weeks. a Cumulative food intake at all points. b Cumulative food intake at 9 weeks after injection. c Body mass at all points. d Body mass 9 weeks after injection. e Food efficiency is expressed as body weight gain per cumulative food intake per week. $\mathbf{f}$ Representative photograph of mice at 8 weeks after injection of the AAV-CTL or the AAVNPGL under a HCD. Each value represents the mean \pm standard error of the mean $(n=$ 8/group). ${ }^{*} P<0.05,{ }^{* *} P<0.01,{ }^{* * *} P<0.005$ vs. AAV-CTL (NC), $+P<0.05,++P<0.01,{ }^{\dagger}+\dagger P$ $<0.005$ vs. AAV-CTL (HCD). NPGL, neurosecretory protein GL; AAV-CTL, AAV-based control vector; AAV-NPGL, AAV-based NPGL-precursor gene vector; NC, normal chow; HCD, high-calorie diet.

\section{Fig. 2.}

Effects of $\mathrm{Npgl}$ overexpression on fat accumulation. The panels show the data obtained by injection of the AAV-CTL or the AAV-NPGL in mice fed NC or a HCD for 9 weeks. a Mass of the inguinal, epididymal, retroperitoneal, and perirenal WAT. b Representative photograph of the iWAT, eWAT, and rWAT in mice fed a HCD. Scale bar $=1 \mathrm{~cm}$. $\mathbf{c}$ Representative photographs in sections of the iWAT in mice fed a HCD. Scale bar $=100 \mu \mathrm{m}$. d Mass of the interscapular BAT. Each value represents the mean \pm standard error of the mean $(\mathrm{n}=8) .{ }^{* * *} P<$ 0.005 vs. AAV-CTL (NC), $+P<0.05,++\dagger P<0.005$ vs. AAV-CTL (HCD). NPGL, neurosecretory protein GL; AAV-CTL, AAV-based control vector; AAV-NPGL, AAV-based NPGL-precursor gene vector; NC, normal chow; HCD, high-calorie diet; WAT, white adipose tissue; iWAT, inguinal WAT; eWAT, epididymal WAT; rWAT, retroperitoneal WAT; BAT, brown adipose tissue. 


\section{Fig. 3.}

Effects of $\mathrm{Npgl}$ overexpression on the muscle and organs. The panels show the data obtained by injection of the AAV-CTL or the AAV-NPGL in mice fed NC or a HCD for 9 weeks. a Mass of the gastrocnemius muscle. b Mass of the testis, liver, kidney, and heart. c Representative photograph of the liver and liver sections stained by Oil Red $\mathrm{O}$ in mice fed a HCD. Scale bars $=1 \mathrm{~cm}$ in the top and $100 \mu \mathrm{m}$ in the bottom. Each value represents the mean \pm standard error of the mean $(\mathrm{n}=8) .{ }^{*} P<0.05,{ }^{* * *} P<0.005$ vs. AAV-CTL (NC), $+++P<0.005$ vs. AAV-CTL (HCD). NPGL, neurosecretory protein GL; AAV-CTL, AAV-based control vector; AAV-NPGL, AAV-based NPGL-precursor gene vector; NC, normal chow; HCD, highcalorie diet.

\section{Fig. 4.}

Effects of $\mathrm{Npgl}$ overexpression on serum parameters. The panels show the data obtained by injection of the AAV-CTL or the AAV-NPGL in mice fed NC or a HCD for 9 weeks. a Serum level of glucose. b Serum level of insulin. c Serum level of triglyceride. d Serum level of free fatty acids. e Serum level of cholesterol. Each value represents the mean \pm standard error of the mean $(\mathrm{n}=7-8) .{ }^{*} P<0.05,{ }^{* *} P<0.005$ vs. AAV-CTL (NC), $+P<0.05,+\dagger+P<0.005$ vs. AAV-CTL (HCD). NPGL, neurosecretory protein GL; AAV-CTL, AAV-based control vector; AAV-NPGL, AAV-based NPGL-precursor gene vector; NC, normal chow; HCD, high-calorie diet.

\section{Fig. 5.}

Effects of $\mathrm{Npgl}$ overexpression on the mRNA expression of lipid metabolism-related genes. The panels show the data obtained by injection of the AAV-CTL or the AAV-NPGL in mice fed NC or a HCD for 9 weeks. a mRNA expression levels in the iWAT. b mRNA expression levels in the liver. Each value represents the mean \pm standard error of the mean $(\mathrm{n}=8) .{ }^{*} P<$ $0.05,{ }^{* *} P<0.01,{ }^{* \star *} P<0.005$ vs. AAV-CTL (NC), $+P<0.05,+++P<0.005$ vs. AAV-CTL (HCD). NPGL, neurosecretory protein GL; AAV-CTL, AAV-based control vector; AAV- 
NPGL, AAV-based NPGL-precursor gene vector; NC, normal chow; HCD, high-calorie diet; WAT, white adipose tissue; iWAT, inguinal WAT.

\section{Fig. 6.}

Effects of $\mathrm{Npgl}$ overexpression on fatty acid ratio in the iWAT. The panels show the data obtained by injection of the AAV-CTL or the AAV-NPGL in mice fed NC or a HCD for 9 weeks. a Ratio of fatty acids (16:1/16:0). b Ratio of fatty acids (18:1/18:0). $\mathbf{c}$ Ratio of fatty acids $(16: 0 / 18: 2 n-6)$. Each value represents the mean \pm standard error of the mean $(\mathrm{n}=8) .{ }^{* * *} P$ $<0.005$ vs. AAV-CTL (NC). NPGL, neurosecretory protein GL; AAV-CTL, AAV-based control vector; AAV-NPGL, AAV-based NPGL-precursor gene vector; NC, normal chow; HCD, high-calorie diet; WAT, white adipose tissue; iWAT, inguinal WAT. 
Table 1. Abbreviation

\begin{tabular}{|c|c|}
\hline Gene & Name \\
\hline$A c c$ & Acetyl-CoA carboxylase \\
\hline Fas & Fatty acid synthase \\
\hline$S c d 1$ & Stearoyl-CoA desaturase 1 \\
\hline Gpat1 & Glycerol-3-phosphate acyltransferase 1 \\
\hline Chrebpa & Carbohydrate-responsive element-binding protein $\alpha$ \\
\hline Cpt1a & Carnitine palmitoyl transferase $1 \mathrm{a}$ \\
\hline Atgl & Adipose triglyceride lipase \\
\hline$H s l$ & Hormone-sensitive lipase \\
\hline$F g f 21$ & Fibroblast growth factor 21 \\
\hline Gapdh & Glyceraldehyde 3-phosphate dehydrogenase \\
\hline Slc2a2 & Solute carrier family 2 member 2 \\
\hline Slc2a4 & Solute carrier family 2 member 4 \\
\hline$C d 36$ & Cluster of differentiation 36 \\
\hline Ppara & Peroxisome proliferator-activated receptor $\alpha$ \\
\hline Ppary & Peroxisome proliferator-activated receptor $\gamma$ \\
\hline Pgcla & Peroxisome proliferator-activated receptor $\gamma$ coactivator $1 \alpha$ \\
\hline $\operatorname{Tnf} \alpha$ & Tumor necrosis factor $\alpha$ \\
\hline Adipoq & Adiponectin \\
\hline Rps 18 & Ribosomal protein S18 \\
\hline$A c t b$ & $\beta$-Actin \\
\hline
\end{tabular}


Table 2. Sequences of oligonucleotide primers for quantitative RT-PCR

\begin{tabular}{|c|c|c|}
\hline Gene & Sense primer (5' to $\left.3^{\prime}\right)$ & Antisense primer (5' to $\left.3^{\prime}\right)$ \\
\hline$A c c$ & TCCGCACTGACTGTAACCACAT & TGCTCCGCACAGATTCTTCA \\
\hline Fas & AGGGGTCGACCTGGTCCTCA & GCCATGCCCAGAGGGTGGTT \\
\hline$S c d 1$ & CTGTACGGGATCATACTGGTTC & GCCGTGCCTTGTAAGTTCTG \\
\hline Gpat1 & TCATCCAGTATGGCATTCTCACA & GCAAGGCCAGGACTGACATC \\
\hline Chrebpa & CGACACTCACCCACCTCTTC & TTGTTCAGCCGGATCTTGTC \\
\hline Cptla & CCTGGGCATGATTGCAAAG & GGACGCCACTCACGATGTT \\
\hline Atgl & AACACCAGCATCCAGTTCAA & GGTTCAGTAGGCCATTCCTC \\
\hline$H s l$ & GCTGGGCTGTCAAGCACTGT & GTAACTGGGTAGGCTGCCAT \\
\hline$F g f 21$ & CCTCTAGGTTTCTTTGCCAACAG & AAGCTGCAGGCCTCAGGAT \\
\hline Gapdh & AAGGTCATCCCAGAGCTGAA & CTGCTTCACCACCTTCTTGA \\
\hline Slc2a2 & GGCTAATTTCAGGACTGGTT & TTTCTTTGCCCTGACTTCCT \\
\hline Slc2a4 & GTAACTTCATTGTCGGCATGG & AGCTGAGATCTGGTCAAACG \\
\hline$C d 36$ & TCCTCTGACATTTGCAGGTCTATC & AAAGGCATTGGCTGGAAGAA \\
\hline Ppara & TCGAATATGTGGGGACAAGG & GACAGGCACTTGTGAAAACG \\
\hline Ppary & GCCCTTTGGTGACTTTATGGA & GCAGCAGGTTGTCTTGGATG \\
\hline Pgcla & GCAACATGCTCAAGCCAAAC & TGCAGTTCCAGAGAGTTCCA \\
\hline $\operatorname{Tnf} \alpha$ & GCCTCTTCTCATTCCTGCTTG & CTGATGAGAGGGAGGCCATT \\
\hline Adipoq & GCAGAGATGGCACTCCTGGA & CCCTTCAGCTCCTGTCATTCC \\
\hline Rps18 & CCTGAGAAGTTCCAGCACAT & TTCTCCAGCCCTCTTGGTG \\
\hline$A c t b$ & GGCACCACACCTTCTACAAT & AGGTCTCAAACATGATCTGG \\
\hline
\end{tabular}



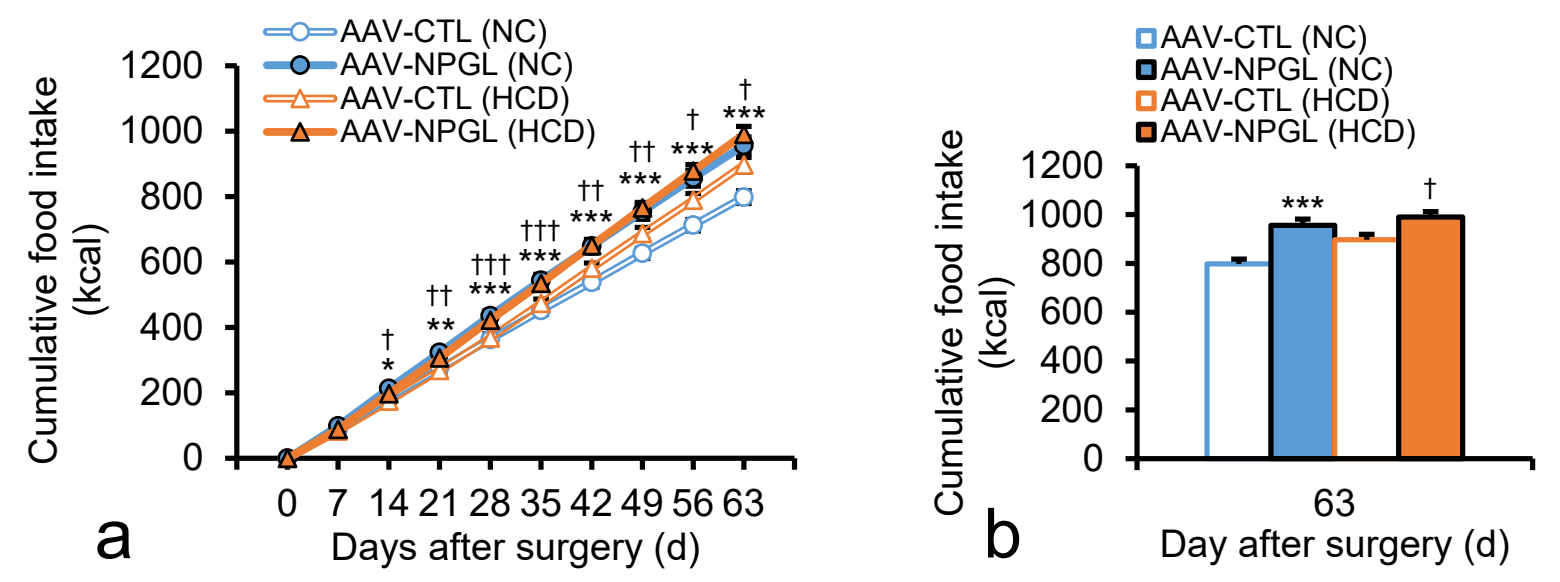

Fig. 1.
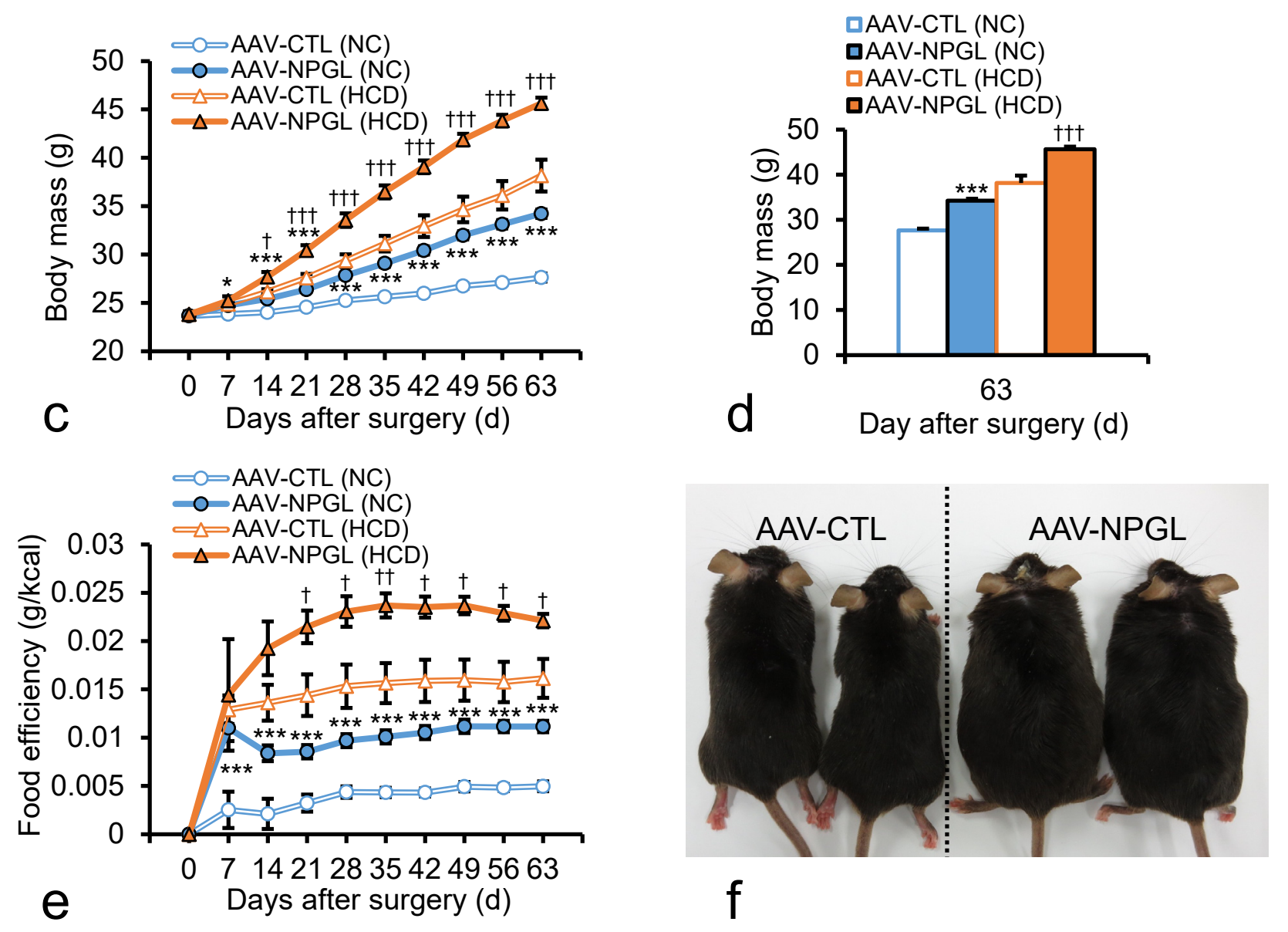
Fig. 2.

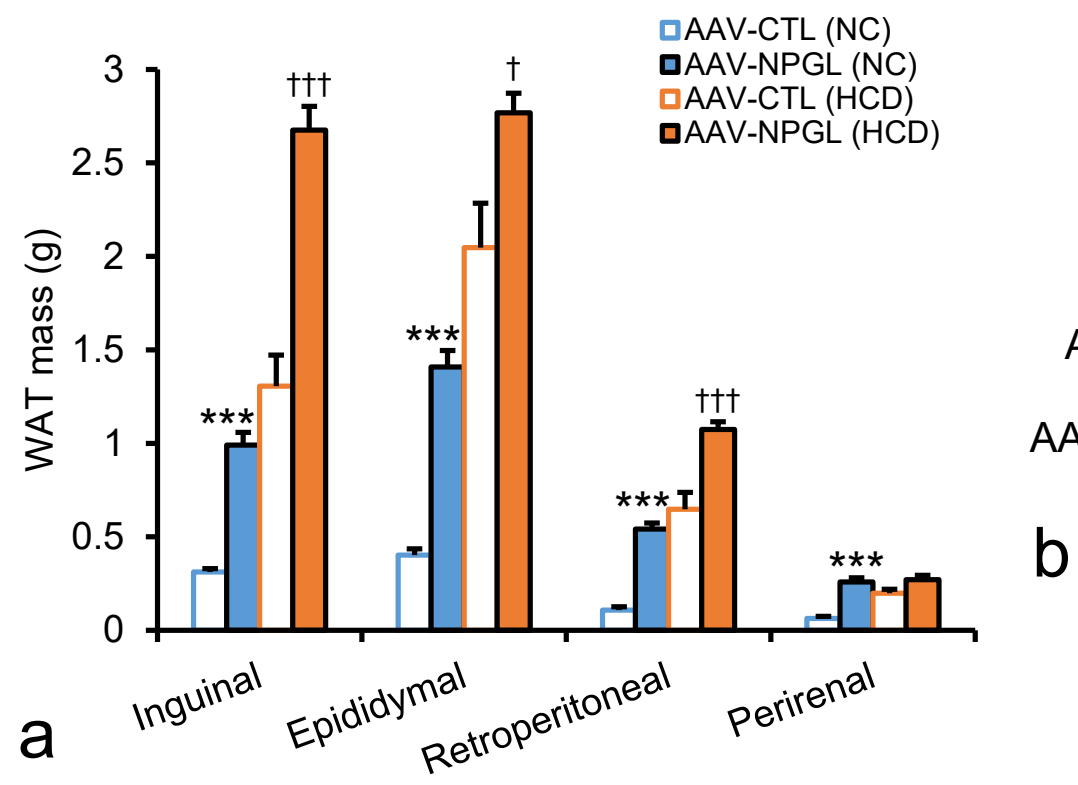

AAV-CTL AAV-NPGL

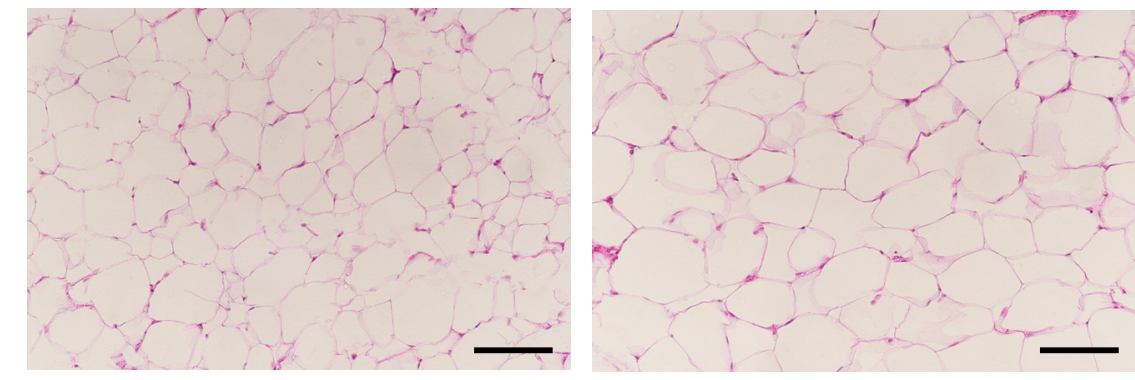

C
IWAT EWAT $\quad$ rWAT

AAV-CTL

AAV-NPGL

b

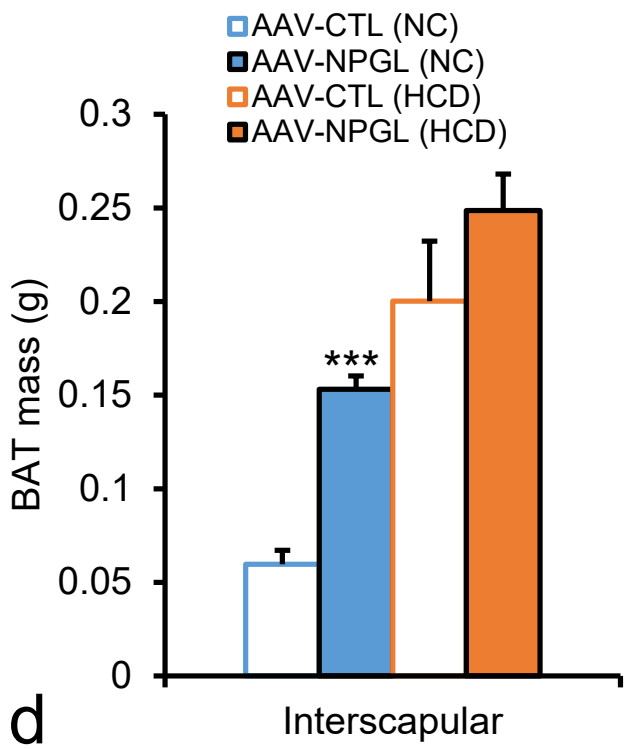


Fig. 3.
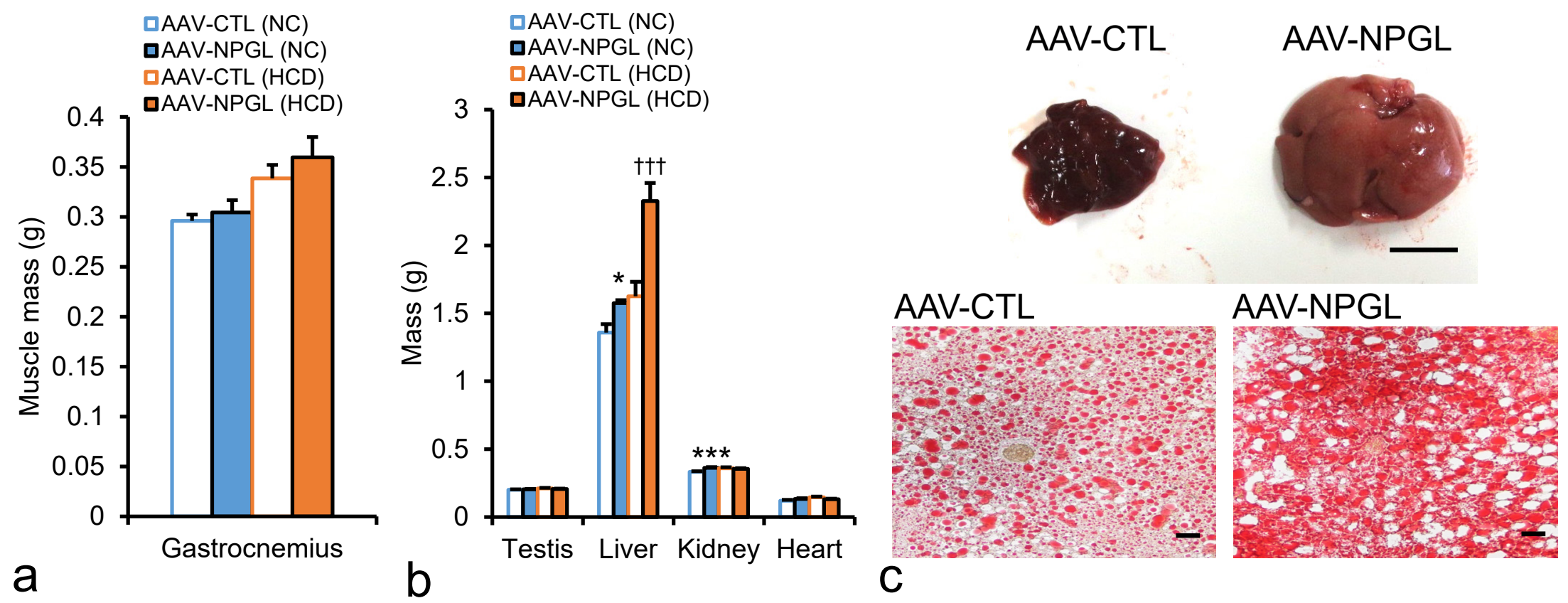


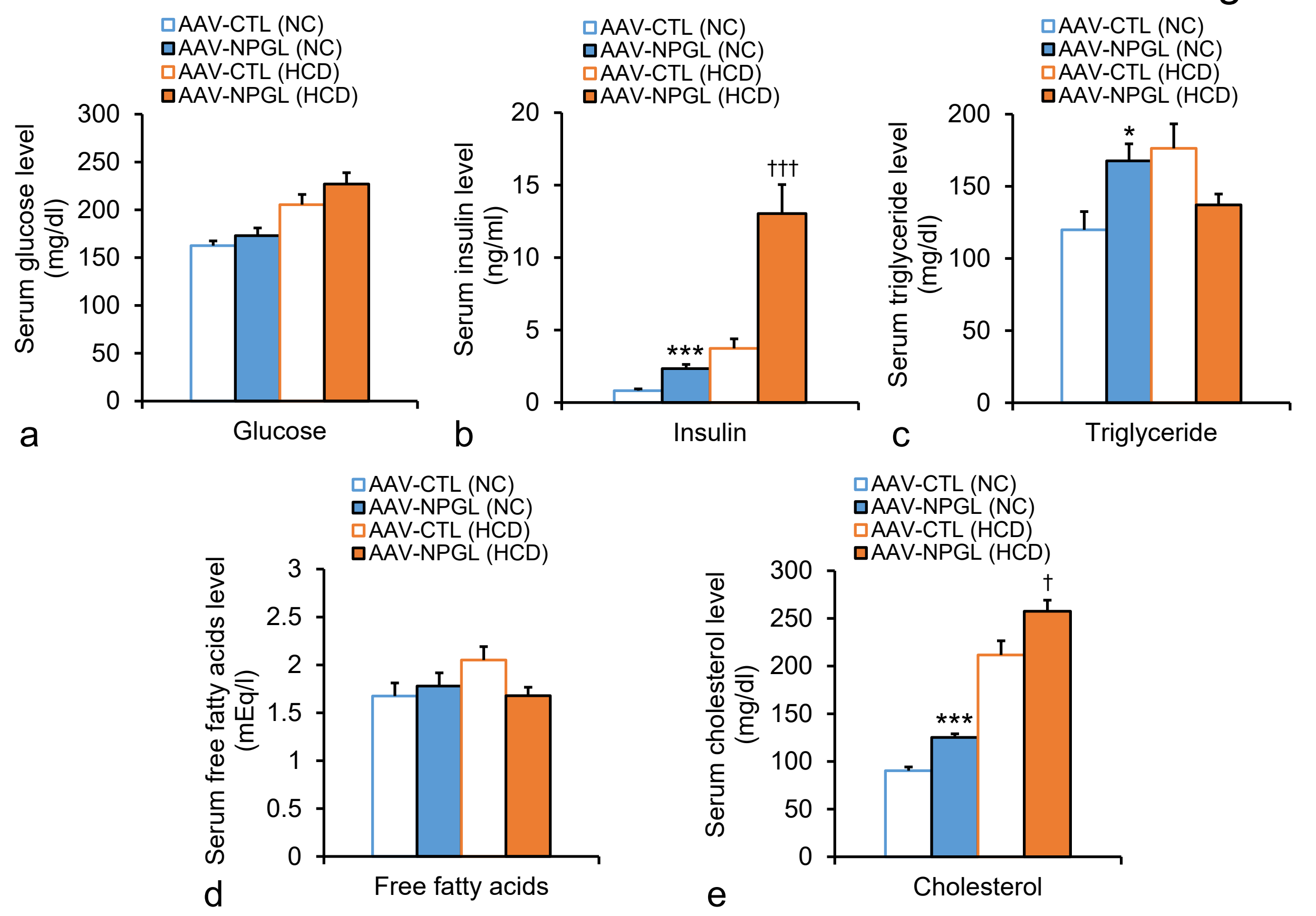




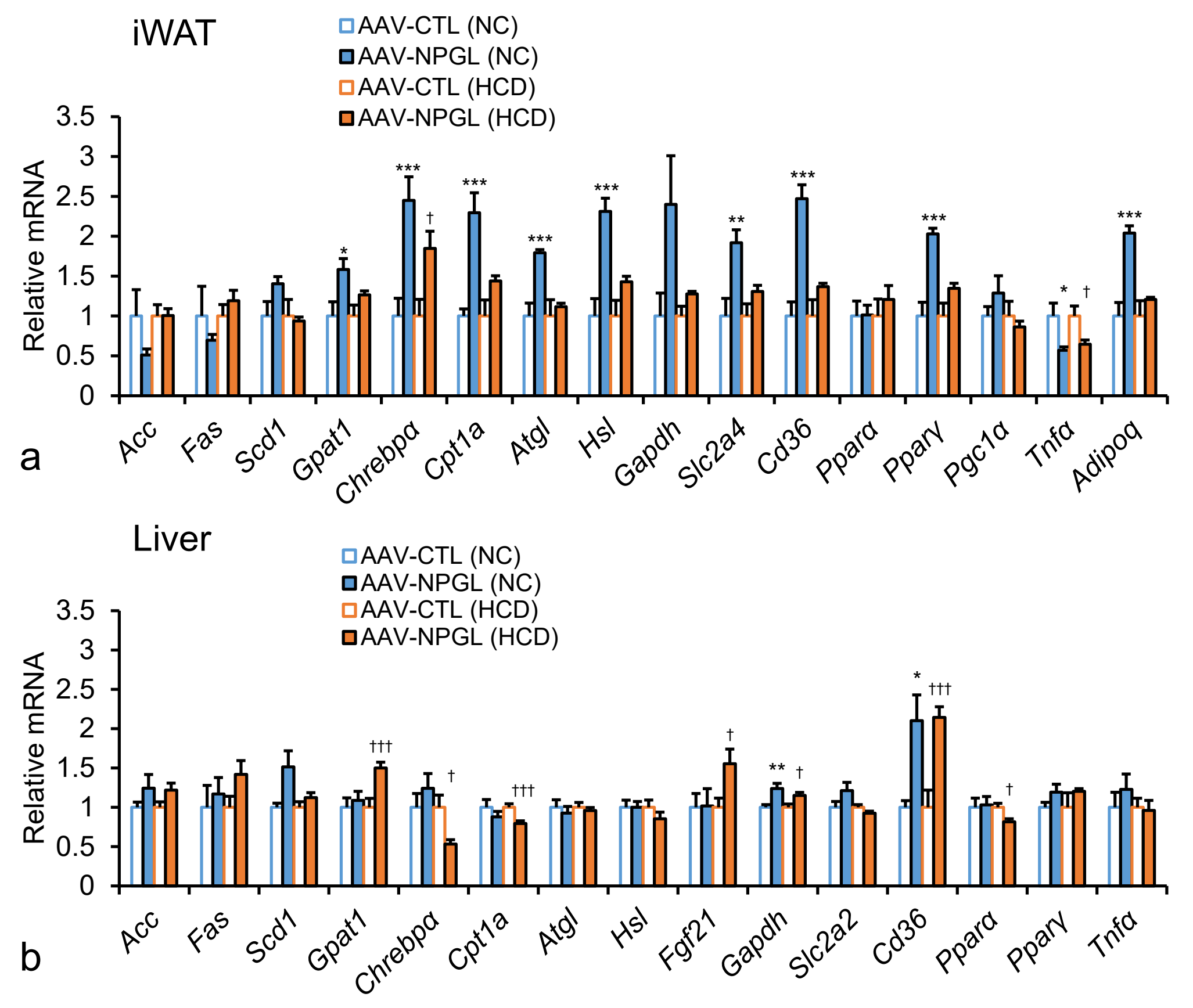

Fig. 5. 
Fig. 6.
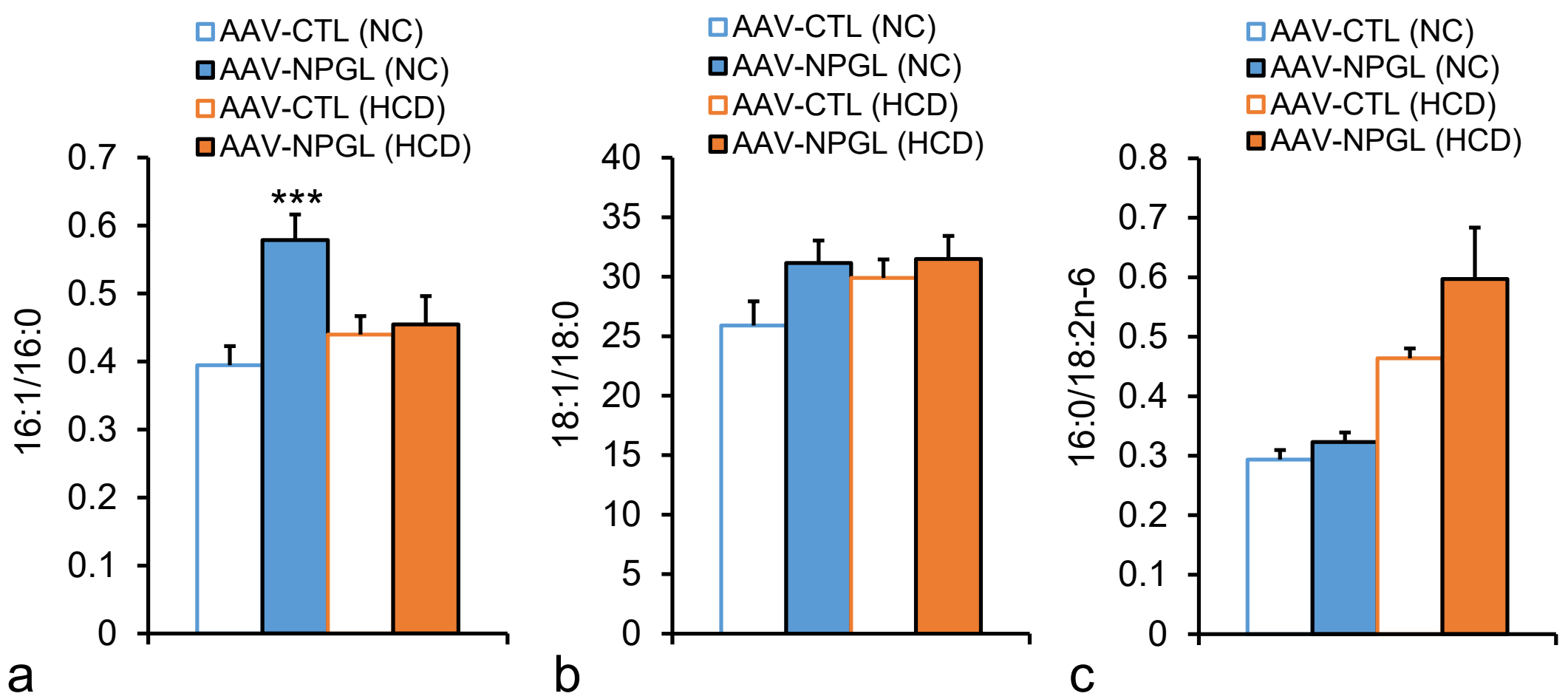